\title{
Efectividad de la intervención educativa en cuidadores de pacientes dependientes en diálisis y valoración de la carga
}

\author{
Antonio Bañobre González \\ Josefa Vázquez Rivera \\ Soledad Outeiriño Novoa \\ Margarita Rodríguez González \\ María González Pascual \\ Julia Graña Álvarez \\ Benita Manso Feijóo \\ Ángeles Estevez Penín \\ $M^{\alpha}$ José Remigio Lorenzo \\ Pilar Álvarez Domínguez \\ Aurora Otero López
}

\author{
Servicio de Nefrología \\ Complexo Hospitalario de Ourense \\ Ourense
}

\begin{abstract}
RESUMEN
Los cambios demográficos y el envejecimiento paulatino de la población con insuficiencia renal crónica en tratamiento renal sustitutivo, así como las múltiples patologías asociadas determinan una población muy dependiente de un cuidador. Por todo esto, evaluamos la carga y grado de conocimientos del cuidador principal de los pacientes "dependientes" en tratamiento renal sustitutivo del Complexo Hospitalario de Ourense. Con los resultados obtenidos realizamos una intervención educativa, valorando posteriormente su efectividad.
\end{abstract}

Se determinó que pacientes son dependientes en su tratamiento y autocuidados y se realizó un estudio de sus cuidadores. Se aplicó la escala sobre carga del cuidador de Zarit y Zarit y la escala sobre conocimientos del cuidador principal, antes y después de la intervención educativa.

CORRESPONDENCIA

Antonio Bañobre González

Servicio de nefrología CHOU

C/ Ramón Puga 54

32005 Ourense
En nuestra población el 24,5\% de los pacientes en HD y el 33,3 \% en DP son dependientes de un cuidador. Los cuidadores no percibían sobrecarga, siendo el grado similar en ambos tipos de diálisis (hospitalaria vs domiciliaria) y además esta percepción disminuía con el tiempo como cuidador. El grado de conocimientos del cuidador aumentó significativamente tras la intervención educativa. Es importante dar una información adecuada al inicio del tratamiento y continuarla a lo largo del mismo, ya que por sí solos los cuidadores no aumentan el nivel de conocimientos.

\section{PALABRAS CLAVE: DEPENDENCIA CUIDADOR INTERVENCIÓN EDUCATIVA TRATAMIENTO RENAL SUSTITUTIVO}

THE EFFECTIVENESS OF EDUCATIONAL INTERVENTION IN CARERS OF PATIENTS IN DIALYSIS AND EVALUATION OF THE LOAD

\section{ABSTRACT}

Good education improves quality of life; the demographic changes and the gradual ageing of the population with chronic renal insufficiency in substitutive renal treatment, and 
the many associated pathologies determine a population that is highly dependent on carers. We therefore evaluate the load and knowledge of the main carers of "dependent" patients in substitutive renal treatment in the Ourense Hospital Complex. With the results, we perform an educational intervention and follow up by evaluating its effectiveness.

It is determined which patients are dependent in their treatment and those who look after themselves, and a study is made of the carers. The Zarit and Zarit scale is applied to the load of the carer and the knowledge of the main carer before and after the education.

In our population, $24.5 \%$ of the patients in $\mathrm{HD}$ and 33.3 $\%$ in DP are dependent on a carer. The carers perceived no overloading, and the load was similar in both types of dialysis (hospital $v$ home), this perception also falling with their time as carers. The carers' knowledge increases significantly following the intervention; suitable information must be given at the beginning of the treatment, and must be continued throughout, as the carers do not increase their knowledge without help.

\section{KEYWORDS:}

\section{DEPENDENCE \\ CARER \\ EDUCATIONAL INTERVENTION \\ SUBSTITUTIVE RENAL TREATMENT}

\section{INTRODUCCIÓN Y OBJETIVOS}

Tanto las características de la enfermedad de los pacientes con insuficiencia renal crónica (IRC) como el tratamiento renal sustitutivo (TRS) provoca una serie de cambios que afectan de manera importante en su vida. La educación sanitaria es una parte integral del rol enfermero, con atención especial a la "prevención, educación y ayuda". Revisando la bibliografía es poca la experiencia publicada referente a cómo se informa a los pacientes y como éstos perciben la información ${ }^{1}$, pero parece claro que la educación paciente/familia reduce el coste causado por las complicaciones de la enfermedad y promueve la comunicación entre profesionales/paciente ${ }^{2}$.

Los cambios demográficos y el envejecimiento paulatino de la población con IRC en TRS así como las múltiples patologías asociadas determinan una población muy dependiente de un cuidador $^{3}$. El cuidador principal considera que desempeñar este trabajo afecta de manera importante a su salud, al uso que hace de su tiempo, a su vida social y a su situación económica 4 .
En nuestro hospital, en los últimos años ha habido un aumento importante de pacientes con IRC que precisan TRS, al igual que ha aumentado la edad media de dichos pacientes. Teniendo en cuenta los dos tipos de TRS claramente diferenciados, los cuidadores principales de los pacientes dependientes, tendrán que aplicar unos cuidados diferentes según el tipo de TRS. Por lo tanto los conocimientos sobre los cuidados a aplicar variarán según el programa de TRS en que estén incluidos.

Por todo esto, nos planteamos detectar los pacientes en TRS del Complexo Hospitalario de Ourense que son dependientes de un cuidador. Una vez identificados, evaluamos la carga percibida y posteriormente el grado de conocimientos de los cuidadores principales de los pacientes dependientes, antes y después de una intervención educativa.

\section{MATERIAL Y MÉTODOS}

Se realiza un estudio cuasiexperimental, desde abril hasta octubre del 2003, para valorar la efectividad de una intervención educativa y la carga en todos los cuidadores de los pacientes "dependientes" en TRS en el Complexo Hospitalario de Ourense.

De los 95 pacientes en TRS en nuestro centro, 53 pacientes reciben HD y 42 realizan DP. Para saber qué pacientes son dependientes de un cuidador principal para su tratamiento y cuidados, se utilizó un criterio de clasificación basado en la siguiente valoración:

- Pacientes en HD: un cuestionario diseñado a tal efecto, con dos preguntas: ¿Quién controla su medicación? Y ante un problema con su fístula arterio-venosa (FAV) o catéter central: ¿Quién toma la decisión de resolver el problema? Se consideró como "dependiente" cuando uno de los dos parámetros era realizado por un cuidador.

- Pacientes en DP: debido a que este tratamiento es realizado por el paciente y/o familia en su domicilio previo a una formación, consideramos como "dependiente" siempre que alguno de los cuidados tiene que ser realizado por un cuidador principal.

Una vez identificados los pacientes que reunían los criterios de inclusión, se contactó telefónicamente con los sus cuidadores principales. Se les explicó el objetivo del estudio, la confidencialidad de los datos y se solicitó su participación voluntaria. Las encuestas (carga y valoración de conocimientos del cuidador), así como los datos demográficos del paciente y cuidador se pasaron individualmente, autoadministradas; exceptuando 
dos cuidadores que por ser analfabetos fueron cumplimentadas con ayuda del personal de enfermería.

\section{Variables del estudio}

Datos demográficos: Se recogen de los pacientes dependientes: edad, sexo, hábitat, estado civil y tiempo en TRS. Del cuidador: tiempo como cuidador, estudios (analfabeto, primarios, secundarios, diplomatura, universitarios) y actitud (positiva, negativa, pasiva).

Valoración de conocimientos del cuidador principal: Se diseña una escala a tal efecto. Esta comprende por 4 bloques de 8 preguntas cada uno, con 4 opciones de respuesta, siendo sólo una válida (Anexos I y II):

- Conocimientos generales sobre la enfermedad del paciente

- Conocimientos generales sobre la dieta

- Conocimientos generales sobre la FAV y sus cuidados o sobre la DP y sus cuidados, según el tipo de TRS

- Conocimientos sobre TRS y farmacológicos

La escala es autoadministrada, antes de la intervención educativa (mayo 2003), y después de la misma (octubre 2003).

\section{Intervención educativa}

Tras la primera valoración de conocimientos, se da a la totalidad de los cuidadores, información oral y escrita sobre los cuatro bloques que se han valorado. Esta intervención educativa es realizada por personal de enfermería de forma estandarizada.

Teniendo en cuenta los datos demográficos de los cuidadores de nuestra población (edad avanzada y nivel de estudios mayoritariamente primarios), se elabora un tríptico informativo de fácil comprensión, reflejando los cuidados principales de la IRC. Esta información le fue entregada a los dos meses de hacer la primera valoración, para dar un refuerzo educativo.

\section{Valoración de la carga del cuidador}

Se aplicó la entrevista sobre la carga del cuidador de "Zarit y Zarit" 1982 5. Dicha escala consta de 22 ítems con rango de respuesta de 0 a 4 . Cuando el rango de respuestas es menor de 46 indica no sobrecarga; 46-56: sobrecarga leve; mayor de 56: sobrecarga intensa.

\section{Análisis estadístico}

Se realizó descriptivo habitual (frecuencia y porcentaje para variables cualitativas; media e intervalos de confianza (IC 95\% para variables cuantitativas). Para comparación de medias se utilizó la U de Man-Whitney o la prueba de Kruskal-Wallis; la correlación entre variables cuantitativas se analizó mediante el coeficiente de Spearman. Para estudiar la efectividad de la intervención se compararon los conocimientos antes y después de la intervención mediante la prueba de Wilcoxon para datos apareados. Se valoran niveles de significación inferiores al 0.05 .

\section{RESULTADOS}

De la valoración para seleccionar los pacientes dependientes de entre los 95 enfermos en TRS en nuestro centro (53 en HD y 42 en DP), 27 resultaron ser dependientes de un cuidador principal. De estos pacientes 12 eran varones y $15 \mathrm{mu}-$ jeres, con un estado civil de 19 casados, 5 solteros y 3 viudos. Vivían 17 en medio rural y 10 en urbano. El tiempo medio en diálisis era de 22,52 meses (IC 95\% 13.96, 31.07). La edad media era de 65,07 años (IC 95\% 59.07, 71.08). Con respecto al TRS de estos pacientes dependientes, 13 recibían HD (edad 73,2; IC 95\% 66.48, 79.98) y 14 recibían DP (edad $57,5 ;$ IC $95 \% 48.99,66.01)(\mathrm{p}=0.005)$.

Tras la selección, los cuidadores fueron citados en la unidad de diálisis (Marzo del 2003). Acudió la totalidad de la muestra excepto 2 cuidadores, debido a problemas de desplazamiento al Hospital, siendo visitados en su domicilio por el personal de enfermería. El tiempo medio como cuidador era de 22,52 meses (IC 95\% 13.96, 31.07), siendo su grado de estudios: 2 analfabetos, 19 primarios, 4 secundarios, y 2 universitarios. La actitud era negativa en sólo 1 y positiva en los otros 26.

Finalizan el estudio 19 cuidadores (12 HD y 7 DP). Ocho cuidadores fueron excluidos de la valoración de conocimientos post-información: uno por cambio de TRS del paciente a su cargo, otro por negativa a continuar en el estudio y 6 por exitus del paciente.

\section{Valoración de conocimientos antes de la información}

Valorando el nivel de conocimientos en los distintos bloques, éstos eran menores en el farmacológico $(3,41 \pm 1,72)$ que en aspectos generales $(5,41 \pm 2,04)$, dieta $(6,04 \pm 1,37)$ y específicos de DP y HD $(6,15 \pm 1,68)$ (tabla 1$)$. Por otra parte, el cuidador que tiene problemas importantes de conocimientos lo tiene de modo uniforme en todos los bloques. En el análisis según el tipo de diálisis (HD vs $\mathrm{DP}$ ) se aprecia que no había diferencias significativas y lo mismo sucedía con el hábitat de los pacientes, en la situación inicial. Referente al tiempo como cuidador un mayor tiempo no se asocia a mayor grado de conocimientos. Y algo similar sucede con el nivel de estudios (tabla 2). 


\begin{tabular}{|c|c|c|c|c|c|c|c|}
\hline & $\begin{array}{c}\text { Bloques } \\
\text { conocimientos }\end{array}$ & & $\mathbf{N}$ & Media & $\begin{array}{l}\text { Desviación } \\
\text { típica }\end{array}$ & $\begin{array}{l}\text { Rango } \\
\text { mínimo }\end{array}$ & $\begin{array}{l}\text { Rango } \\
\text { máximo }\end{array}$ \\
\hline \multirow{4}{*}{$\begin{array}{l}\text { Antes de la } \\
\text { intervención } \\
\text { Educativa }\end{array}$} & $\begin{array}{c}\text { Conocimientos } \\
\text { generales }\end{array}$ & $\begin{array}{l}\text { H.D. } \\
\text { D.P. } \\
\text { Total }\end{array}$ & $\begin{array}{l}13 \\
14 \\
27\end{array}$ & $\begin{array}{l}5,85^{*} \\
5,00^{*} \\
5,41^{* *}\end{array}$ & $\begin{array}{l}2,08 \\
2,00 \\
2,04\end{array}$ & $\begin{array}{l}2 \\
2 \\
2\end{array}$ & $\begin{array}{l}8 \\
8 \\
8\end{array}$ \\
\hline & Dieta & $\begin{array}{l}\text { H.D. } \\
\text { D.P. } \\
\text { Total }\end{array}$ & $\begin{array}{l}13 \\
14 \\
27\end{array}$ & $\begin{array}{l}6,54^{*} \\
5,57^{*} \\
6,04^{* *}\end{array}$ & $\begin{array}{l}1,05 \\
1,50 \\
1,37\end{array}$ & $\begin{array}{l}5 \\
2 \\
2\end{array}$ & $\begin{array}{l}8 \\
7 \\
8\end{array}$ \\
\hline & Conocimientos TRS & $\begin{array}{l}\text { H.D. } \\
\text { D.P. } \\
\text { Total }\end{array}$ & $\begin{array}{l}13 \\
14 \\
27\end{array}$ & $\begin{array}{l}5,69^{*} \\
6,57 \\
6,15^{*}\end{array}$ & $\begin{array}{l}1,80 \\
1,50 \\
1,68\end{array}$ & $\begin{array}{l}1 \\
4 \\
1\end{array}$ & $\begin{array}{l}8 \\
8 \\
8\end{array}$ \\
\hline & $\begin{array}{l}\text { Tratamiento } \\
\text { farmacológico }\end{array}$ & $\begin{array}{l}\text { H.D. } \\
\text { D.P. } \\
\text { Total }\end{array}$ & $\begin{array}{l}13 \\
14 \\
27\end{array}$ & $\begin{array}{c}3,31 \\
3,50^{* * *} \\
3,41^{*}\end{array}$ & $\begin{array}{l}2,02 \\
1,45 \\
1,72\end{array}$ & $\begin{array}{l}0 \\
1 \\
0\end{array}$ & $\begin{array}{l}8 \\
7 \\
8\end{array}$ \\
\hline \multirow{4}{*}{$\begin{array}{l}\text { Después } \\
\text { de la } \\
\text { intervención } \\
\text { Educativa }\end{array}$} & $\begin{array}{c}\text { Conocimientos } \\
\text { generales }\end{array}$ & $\begin{array}{l}\text { H.D. } \\
\text { D.P. } \\
\text { Total }\end{array}$ & $\begin{array}{c}12 \\
7 \\
19\end{array}$ & $\begin{array}{l}7,17^{*} \\
7,00^{*} \\
7,11^{* *}\end{array}$ & $\begin{array}{l}1,19 \\
0,82 \\
1,05\end{array}$ & $\begin{array}{l}5 \\
6 \\
5\end{array}$ & $\begin{array}{l}8 \\
8 \\
8\end{array}$ \\
\hline & Dieta & $\begin{array}{l}\text { H.D. } \\
\text { D.P. } \\
\text { Total }\end{array}$ & $\begin{array}{c}12 \\
7 \\
19\end{array}$ & $\begin{array}{l}7,58^{*} \\
7,14^{*} \\
7,42^{* *}\end{array}$ & $\begin{array}{l}0,67 \\
0,90 \\
0,77\end{array}$ & $\begin{array}{l}6 \\
6 \\
6\end{array}$ & $\begin{array}{l}8 \\
8 \\
8\end{array}$ \\
\hline & Conocimientos TRS & $\begin{array}{l}\text { H.D. } \\
\text { D.P. } \\
\text { Total }\end{array}$ & $\begin{array}{c}12 \\
7 \\
19\end{array}$ & $\begin{array}{l}7,00^{* * *} \\
7,71 \\
7,26^{*}\end{array}$ & $\begin{array}{l}0,95 \\
0,76 \\
0,93\end{array}$ & $\begin{array}{l}5 \\
6 \\
5\end{array}$ & $\begin{array}{l}8 \\
8 \\
8\end{array}$ \\
\hline & $\begin{array}{c}\text { Tratamiento } \\
\text { farmacológico }\end{array}$ & $\begin{array}{l}\text { H.D. } \\
\text { D.P. } \\
\text { Total }\end{array}$ & $\begin{array}{c}12 \\
7 \\
19\end{array}$ & $\begin{array}{l}3,75 \\
6,00^{*} \\
4,58^{*}\end{array}$ & $\begin{array}{l}1,71 \\
1,83 \\
2,04\end{array}$ & $\begin{array}{l}2 \\
3 \\
2\end{array}$ & $\begin{array}{l}7 \\
8 \\
8\end{array}$ \\
\hline
\end{tabular}

(TRS: Tratamiento renal sustitutivo)

$*\left(\begin{array}{llll}\text { p } & 0.05) \quad * * & \text { (p } 0.005)\end{array}\right.$

Tabla 1. Valoración de conocimientos antes y después de intervención educativa, total y según tipo de TRS.

Comparación del nivel de conocimientos antes y después de la información

Comparando el valor medio antes y después de la información en los distintos bloques de conocimientos, las diferencias son estadísticamente significativas, comparando cada cuidador consigo mismo. El nivel de cocimientos del cuidador después de la información aumentó en todos los bloques: Conocimientos generales $(7,11 \pm 1,05)$, Dieta $(7,42 \pm 0,77)$, Tratamiento sustitutivo $(7,26 \pm 0,93)$, Tratamiento farmacológico $(4,58 \pm 2,04)$.

Según el tipo de TRS, antes y después de la información, encontramos que los cuidadores de pacientes dependientes en HD mejoraron sus conocimientos de forma significativa en los bloques de: Conocimientos generales $\mathrm{p}=0,018$; Dieta $p=0,016 ; y$ Tratamiento sustitutivo $p=0,040$. No encontrando diferencia significativa en el bloque de conocimientos de Tratamiento farmacológico. Los cuidadores de pacientes dependientes en DP mejoraron sus conocimientos de forma significativa en los bloques de: Conocimientos generales $p=0,066$; Dieta $p=0,039$ y Tratamiento farmacológico $p=0,046$. No encontrando diferencia significativa en el bloque de conocimientos de Tratamiento sustitutivo.

\section{Valoración de la carga percibida}

Dos cuestionarios no fueron debidamente cumplimentados, por lo que el número de cuidadores principales sobre los que se ha realizado esta valoración es de 25 . Tras la aplicación de la Entrevista de "Zarit y Zarit" se observó que la carga percibida por el cuidador tenía un valor de $30.7 \pm 22.5$; 20 cuida- 
dores no percibían sobrecarga (80\%), 2 percibían sobrecarga leve (8\%) y 3 sobrecarga intensa (12\%). Analizando los posibles factores que podrían influenciarla, se aprecia que no hay diferencia significativa de la carga percibida con independencia del tipo de TRS, lugar de residencia, ni nivel de estudios (tabla 3). Valorando el tiempo como cuidador si hay diferencia significativa, pues al aumentar el tiempo como cuidador por encima de los 60 meses, disminuye la carga percibida $(\mathrm{p}=0.02)$ (tabla 3).

\begin{tabular}{|c|c|c|c|}
\hline & & Número & Rango Promedio \\
\hline \multirow{6}{*}{$\begin{array}{l}\text { Conocimientos } \\
\text { Generales }\end{array}$} & Analfabeto & 2 & 14.00 \\
\hline & Primarios & 19 & 11.71 \\
\hline & Secundarios /FP1 & 4 & 20.50 \\
\hline & Diplomatura /FP2 & 1 & 25.50 \\
\hline & Universitarios & 1 & 20.00 \\
\hline & Total & 27 & \\
\hline \multirow{6}{*}{ Dieta } & Analfabeto & 2 & 13.25 \\
\hline & Primarios & 19 & 12.92 \\
\hline & Secundarios /FP1 & 4 & 14.88 \\
\hline & Diplomatura /FP2 & 1 & 26.00 \\
\hline & Universitarios & 1 & 20.50 \\
\hline & Total & 27 & \\
\hline \multirow{6}{*}{$\begin{array}{l}\text { Conocimientos } \\
\text { sobre FAV o DP }\end{array}$} & Analfabeto & 2 & 6.25 \\
\hline & Primarios & 19 & 12.61 \\
\hline & Secundarios /FP1 & 4 & 21.00 \\
\hline & Diplomatura /FP2 & 1 & 24.50 \\
\hline & Universitarios & 1 & 17.50 \\
\hline & Total & 27 & \\
\hline \multirow{6}{*}{$\begin{array}{l}\text { Conocimientos TRS } \\
\text { y Farmacológico }\end{array}$} & Analfabeto & 2 & 6.50 \\
\hline & Primarios & 19 & 12.89 \\
\hline & Secundarios /FP1 & 4 & 16.75 \\
\hline & Diplomatura /FP2 & 1 & 27.00 \\
\hline & Universitarios & 1 & 26.00 \\
\hline & Total & 27 & \\
\hline
\end{tabular}

(DP: diálisis peritoneal; FAV: fístula arteriovenosa; TRS: tratamiento renal sustitutivo) Tabla 2. Valoración conocimientos del cuidador principal según nivel de estudios

\section{DISCUSIÓN}

La educación sanitaria es una parte integral del rol enfermero, con atención especial a la "prevención, educación y ayuda". Una buena educación mejora la calidad de vida, mientras que por el contrario, la falta de información adecuada provoca un efecto negativo. La European Dialysis and Transplant Nurses

\begin{tabular}{|l||l||c|c|}
\hline \multirow{2}{*}{ Tipo de TRS } & & Media & Desviación Standard \\
\hline \multirow{3}{*}{ Lugar de Residencia } & H.D & 36.00 & 29.65 \\
\cline { 2 - 4 } & D.P. & 27.36 & 15.27 \\
\cline { 2 - 4 } & Rural & 28.13 & 25.41 \\
\hline \multirow{4}{*}{ Nivel de Estudios } & Unbano & 34.60 & 17.93 \\
\cline { 2 - 4 } & Analfabeto & 15.50 & 19.09 \\
\cline { 2 - 4 } & Srimarios & 31.06 & 22.72 \\
\cline { 2 - 4 } & Secundarios/FP1 & 31.50 & 24.20 \\
\cline { 2 - 4 } & Diplomatura/FP2 & 65 & \\
\cline { 2 - 4 } & Universitarios & 18 & \\
\hline \multirow{3}{*}{$\begin{array}{l}\text { Tiempo como } \\
\text { Cuidador }\end{array}$} & $<=60$ meses & 38.73 & $25.17^{*}$ \\
\cline { 2 - 4 } & $>60$ meses & 18.70 & $10.03^{*}$ \\
\hline
\end{tabular}

(TRS: tratamiento renal sustitutivo, HD: hemodiálisis; DP: diálisis peritoneal). * $\mathrm{p}=0.02$

Tabla 3. Valoración de la carga percibida por el cuidador principal.

Association European Renal Care Association (EDTNA / ERCA) lo incluye en el punto de vista de su filosofía 6 . Por ello una de las metas de los profesionales de la enfermería en pacientes con patologías crónicas es conseguir que ellos y sus familias tengan un nivel adecuado de conocimientos sobre su enfermedad y sus cuidados ${ }^{7}$. Por lo que estudios sobre la valoración de la carga percibida y la efectividad de una intervención educativa de los cuidadores de pacientes dependientes en TRS pueden facilitar los cuidados.

En nuestro centro casi un 30\% de los pacientes, con independencia del sexo, resultaron ser dependientes de un cuidador principal. Dadas las características de nuestra población, había más pacientes de medio rural que urbano, con predominio de pacientes casados. Si bien existen diferencias cualitativas en cuanto a los tipos diferentes de TRS, una hospitalaria y la otra domiciliaria, no había diferencias importantes en el porcentaje de dependientes según el tipo de diálisis. Sin embargo es de resaltar la importante diferencia de edad entre los pacientes en $\mathrm{HD}$ versus $\mathrm{DP}$ ( 73 vs 57,5 años).

Tanto las características de la enfermedad de los pacientes con IRC como su TRS provoca una serie de cambios que afectan de manera importante en su vida, ejerciendo un gran impacto en su vida social y familiar. La Sociedad Española de Enfermería Nefrológica (SEDEN), a través del Manual de Protocolos de Actuación de Enfermería Nefrológica, establece las actividades y recomendaciones necesarias para dar al paciente y familia la información adecuada ${ }^{8}$. Por otra parte, parece evidente que la educación paciente/familia reduce el coste causado por sus complicaciones y promueve la comunicación entre profesionales y pacientes ${ }^{2}$. Las publicaciones relacionadas con el proceso de información al paciente con IRC hacen referencia a la importancia de la familia en la participación de 
dicho proceso ${ }^{9-11}$. En este estudio se aprecia un déficit de conocimientos en los cuidadores principales. Si bien este déficit era mayor en los aspectos farmacológicos, era también relevante en aspectos básicos del cuidado y dieta. Por otra parte esta falta de conocimientos no era diferente según el tipo de diálisis, ni el medio rural o urbano. Aunque parezca sorprendente la ausencia de significación estadística entre el nivel de estudios y el grado de conocimientos, la mayoría habían cursado sólo primarios, lo que justificaría este hallazgo. Es destacable que un mayor tiempo como cuidador no se asocia a un mayor grado de conocimientos, lo que resalta la importancia de una educación temprana y continuada.

Teniendo en cuenta los resultados obtenidos, podemos decir que la intervención educativa ha sido efectiva ya que comparando cada cuidador consigo mismo antes y después de la intervención, la diferencia fue estadísticamente significativa; por lo que parece razonable pensar que si aplicamos la intervención a otra muestra poblacional con características similares se obtendrían los mismos resultados.

En diferentes estudios se ha visto que el familiar cuidador conlleva una grave carga social y psicológica que recae básicamente en la mujer-hija ${ }^{12}$. En general, el cuidador principal considera que desempeñar este trabajo ha afectado de manera importante a su salud, al uso que hace de su tiempo, a su vida social y a su situación económica ${ }^{4}$. Sin embargo en nuestra población estudiada, la carga percibida era baja y no estaba influenciada por el tipo de TRS, lugar de residencia ni nivel de estudios. Si bien con respecto al nivel de estudios del cuidador principal puede apreciarse una tendencia hacia una asociación entre mayor nivel de estudios con mayor carga percibida. Por otra parte, el mayor tiempo como cuidador disminuye aún más la carga percibida, esto podría deberse a que con el tiempo el cuidador principal asume su nuevo rol, ya que no se asocia a un aumento de conocimientos.

En conclusión, en nuestra población en TRS el 24,5\% de los pacientes en HD y el 33,3\% en DP son dependientes de un cuidador. Los cuidadores no percibían sobrecarga, siendo el grado similar en ambos tipos de TRS (HD vs DP) y además disminuía con el aumento de tiempo como cuidador. Se observa un déficit de conocimientos en los cuidadores antes de la información, aumentando estos significativamente después de la intervención educativa. Es importante dar una información adecuada al inicio del TRS y continuarlo a lo largo del mismo, ya que por si solos los cuidadores no aumentan el nivel de conocimientos.

\section{Agradecimientos}

Al Departamento de Investigación por su colaboración y a todo el personal del Servicio de Nefrología del CHOU.

\section{BIBLIOGRAFÍA}

1. Hayslip DM and Suttle CD. Pre-ESRD patient education: A review of the literature. Advances in Renal Replacement Theraphy 1995; 2 (3): 217-226.

2. Pascual R, Andreu L. Programa de educación sanitaria del paciente en hemodiálisis. Libro de Comunicaciones del XV Congreso de la SEDEN. Bilbao. 1990.

3. Valderrábano F. Tratado de Hemodiálisis. Barcelona: Editorial Medica; 1999.

4. Mateo Rodriguez I, Millán Carrasco A, García Callvante M, Gutierrez Cuadra P, Gonzalo Jimenez E, López Fernández LA: Cuidadores familiares de personas con enfermedad neurodegenerativa: perfil, aportaciones e impacto de cuidar. Atención Primaria 2000; 26( 3):139- 144.

5. Izal y Montorio. Evaluación del medio y del cuidador del demente. En: Ser T, Peña J (eds). Evaluación neuropsicológica y funcional de la Demencia. Barcelona: Prous; 1994.

6. EDTNA/ERCA: European Core Curriculum for a Post-Basic Course in Nephrology Nursing. 1994.

7. Vázquez Rivera J, Vázquez Pérez A, Martínez Campos J, Castiñeiras Iglesias MJ. Conocimientos básicos del paciente en programa de hemodiálisis. "Valoración de enfermería". Libro de Comunicaciones del XXII Congreso SEDEN. Santander. 1997.

8. Ronda García. Manual de Protocolos de Actuación en Enfermería Nefrológica. SEDEN. 1996.

9. Benavides MA, Gonzalez MP, Ballardo A. Proceso de educación sanitaria en pacientes con IRC en pre-diálisis. III Seminario Español de la EDTNA/ERCA. Segovia. 1993.

10. Miguel M, Castro N y col. Importancia de la información que reciben los pacientes antes de iniciar hemodiálisis periódica. Libro de comunicaciones del XVIII Congreso de la SEDEN. La Coruña. 1993.

11. De Eusebio Y, Castro MJ, Celadilla O y col. Consulta de enfermería en la unidad de CAPD. Libro de Comunicaciones del XVI Congreso de la SEDEN, Madrid. 1991.

12. Puig C, Hernández Monsalve M, Gervás J. Los enfermos crónicos recluidos en su domicilio y su repercusión en la familia: una investigación cualitativa. Atención Primaria 1992; 10(1):550-552. 


\section{ANEXO I. CUESTIONARIO EVALUACIÓN DE CONOCIMIENTOS PARA CUIDADORES DE PACIENTES DEPENDIENTES EN DIALISIS PERITONEAL}

\section{CONOCIMIENTOS VARIOS}

1. ¿Sabe usted porque esta incluido en programa de diálisis?
a. Para recuperar su función renal.
b. Porque su función renal es inferior a un $10 \%$.
c. Para aumentar su diuresis.
d. No sabe.

2. ¿Puede usted viajar o irse de vacaciones mientras esta en programa de diálisis?
a. Nunca
b. Si. Su equipo de profesionales le organizará los tratamientos
poniéndose en contacto y concertando los mismos con uni- dades de diálisis de otras ciudades
c. Si. Puede dejar de dializarse unos días.
d. No sabe.

3. ¿Con quien debe ponerse en contacto si le surge algún problema?
a. Con su familia.
b. Con su médico de cabecera.
c. Con su unidad de diálisis, o servicio de urgencias de un cen- tro hospitalario.
d. No sabe.

4. ¿Cree usted que puede trasplantarse cuando quiera?
a. No hay riñones para todos, existe una lista de espera para ello.
b. Si.
c. Depende del tiempo que lleve en programa de diálisis.
d. No sabe.

5. ¿Qué es ALCER?
a. Un club recreativo.
b. Asociación de enfermos renales.
c. Asociación socio-cultural
d. No sabe.

6. ¿Sabe a quien puede dirigirse si tiene algún problema socio-económico?
a. Al sacerdote de su parroquia
b. Al asistente social y/o ALCER
c. A sus compañeros de diálisis.
d. No sabe.

7. Si por algún motivo no puede seguir en programa de diálisis. ¿conoce otras alternativas?
a. No existe ninguna otra técnica de diálisis.
b. Si la hemodiálisis.
c. La única alternativa es el trasplante.
d. No sabe.

8. Si ante cualquier problema de salud ajeno a su patología renal le recetan un tratamiento.
a. Lo tomará como le indiquen.
b. Consultará previamente con el nefrólogo.
c. No lo tomará
d. No sabe.

DIETA

1. ¿Por qué es importante la dieta?
a. No es importante.
b. Porque determinados alimentos pueden aportar sustancias dañinas para el organismo en el periodo de interdiálisis.
c. Porque una dieta adecuada puede recuperarle la función re- nal.
d. No sabe.

2. ¿Sabe porque son peligrosos los alimentos que contienen potasio?
a. Porque producen picor.
b. Porque producen dolor muscular, parada cardiaca, muerte...
c. Porque producen pérdida de audición.
d. No sabe.

3. ¿Qué alimento contiene más potasio?
a. Verduras y frutas.
b. Leche.
c. Pastas.
d. No sabe.

4. Las verduras son alimentos ricos en potasio. ¿Cómo deben de tomarse para disminuir el nivel de potasio?
a. Crudas.
b. Cocidas.
c. Cocidas en dos aguas.
d. No sabe.

5. ¿Cuántas piezas de fruta puede tomar al día?
a. Ninguna.
b. Una.
c. Todas las que quiera.
d. No sabe.

6. ¿Cuánto líquido puede ingerir en su dieta diaria?
a. Libre.
b. $500 \mathrm{cc}+$ diuresis.
c. Dos litros.
d. No sabe.

7. ¿Qué ocurre cuando ingiere mucho líquido?
a. Respira mal, hinchazón de manos y piernas.
b. Tiene calambres.
c. Orina más.
d. No sabe.

8. ¿Por qué debe de comer sin sal?
a. Para tener menos sed y para que su tensión arterial no se al- tere.
b. Porque se disminuye el tiempo de diálisis.
c. Porque orina más.
d. No sabe. 


\section{DIÁLISIS PERITONEAL}

1. ¿Que es un catéter de diálisis peritoneal?

a. Un tubo blando que se coloca en abdomen

b. Unión de una arteria y una vena

c. Un tubo que se coloca en vena femoral

d. No sabe.

2. ¿Para que sirve el catéter de diálisis peritoneal?
a. Para que entre y salga el líquido de diálisis
b. Para eliminar líquidos
c. Para controlar peso
d. No sabe

3. La cura del catéter es imprescindible realizarla:
a. Todos los días
b. Un día por semana
c. Tres veces al día
d. No sabe

4. ¿Como debe realizar la cura del catéter de diálisis peritoneal?
a. Limpiar con suero fisiológico, secar con gasa estéril, colocar gasa alrededor catéter y colocar apósito estéril.
b. Lavar con agua, secar con gasa estéril y colocar apósito es- téril.
c. a y b son ciertas
d. No sabe

5. ¿Que debe hacer si el orificio supura y/o está enrojecido?
a. Acudirá al médico de cabecera
b. Se pondrá en contacto con centro de diálisis
c. Esperar unos días a ver si se resuelve
d. No sabe

6. ¿Que debe hacer si el líquido no entra o no sale al realizar el intercambio de diálisis?

a. Comprobar que las pinzas del sistema están abiertas y/o no haya acodamientos; $y$ si no se resuelve ponerse en contacto con el centro de diálisis

b. No hacer intercambio y esperar al siguiente

c. Llamar al 061

d. No sabe

7. Ante rotura o desconexión accidental del catéter de diálisis ¿ que debe hacer?
a. Acudir a centro de salud
b. Intentar solucionarlo y no avisar
c. Acudir rápidamente a centro de diálisis
d. No sabe

8. ¿Que debe hacer si al drenar el liquido es turbio y/o tiene dolor abdominal?

a. Introducir antibióticos en bolsa

b. Esperar al día siguiente a ver si el líquido es más claro y/o cede el dolor

c. Acudir a centro de diálisis

d. No sabe

\section{TRATAMIENTO SUSTITUTIVO / FARMACOLÓGICO}

1. La diálisis peritoneal consiste:

a. En introducir un líquido especial en cavidad abdominal a través de un catéter

b. Es un método por el que se eliminan toxinas y exceso de líquidos, utilizando el peritoneo como filtro

c. a y b son ciertas

d. No sabe

2. En la diálisis peritoneal continua ambulatoria se realizan:

a. 4 o 5 intercambios manuales, en casa durante el día y todos los días.

b. 4 intercambios en casa descansando los fines de semana

c. 4 intercambios en casa, durante la noche

d. No sabe

3. ¿Que es la diálisis peritoneal automática?
a. Diálisis que se realiza en casa, por la noche y con ayuda de una máquina
b. El tratamiento se realiza todas las noches
c. a y b son ciertas
d. No sabe

4. ¿Sabe como evitar la entrada de gérmenes al realizar los intercambios de diálisis?

a. Realizando los intercambios con las ventanas abiertas

b. Utilizando mascarilla y desinfectando manos

c. Utilizando para secado de manos, toalla usada por otra persona

d. No sabe

5. ¿Que debe hacer si tiene sobrecarga de líquidos?
a. Tomar algo para orinar más
b. Dejar de tomar líquidos totalmente esperando que se resuel- va el problema
c. Ponerse en contacto con centro de diálisis
d. No sabe

6. ¿Sabe para que sirve la inyección de nesp que se administra en su domicilio?
a. Para aumentar el calcio de los huesos
b. Para corregir anemia y evitar transfusiones
c. Para regular tensión arterial y frecuencia cardíaca
d. No sabe

7. En relación al fósforo:
a. Suele aumentar en la IRC y puede producir picor
b. Disminuye evitando alimentos ricos en fósforo, con la diálisis y con la medicación
c. a y b son ciertas
d. No sabe

8. ¿Sabe como debe tomar el renagel si lo tiene pautado en su tratamiento?
a. Al comienzo de las comidas
b. Después de las comidas
c. En medio de las comidas
d. No sabe 


\section{ANEXO II. CUESTIONARIO DE EVALUACIÓN DE CONOCIMIENTOS PARA CUIDADORES DE PACIENTES DEPENDIENTES EN HEMODIALISIS}

\section{CONOCIMIENTOS VARIOS}

1. ¿Sabe usted porque esta incluido en programa de diálisis?
a. Para recuperar su función renal.
b. Porque su función renal es inferior a un $10 \%$.
c. Para aumentar su diuresis.
d. No sabe.

2. ¿Puede usted viajar o irse de vacaciones mientras esta en programa de diálisis?

a. Nunca

b. Si. Su equipo de profesionales le organizará los tratamientos poniéndose en contacto y concertando los mismos con unidades de diálisis de otras ciudades

c. Si. Puede dejar de dializarse unos días.

d. No sabe.

3. ¿Con quien debe ponerse en contacto si le surge algún problema?
a. Con su familia.
b. Con su médico de cabecera.
c. Con su unidad de diálisis, o servicio de urgencias de un cen- tro hospitalario.
d. No sabe.

4. ¿Cree usted que puede trasplantarse cuando quiera?
a. No hay riñones para todos, existe una lista de espera para ello.
b. Si.
c. Depende del tiempo que lleve en programa de diálisis.
d. No sabe.

5. ¿Qué es ALCER?
a. Un club recreativo.
b. Asociación de enfermos renales.
c. Asociación socio-cultural
d. No sabe.

6. ¿Sabe a quien puede dirigirse si tiene algún problema socio-económico?
a. Al sacerdote de su parroquia
b. Al asistente social y/o ALCER
c. A sus compañeros de diálisis.
d. No sabe.

7. Si por algún motivo no puede seguir en programa de diálisis. ¿conoce otras alternativas?
a. No existe ninguna otra técnica de diálisis.
b. Si la diálisis peritoneal
c. La única alternativa es el trasplante.
d. No sabe.

8. Si ante cualquier problema de salud ajeno a su patología renal le recetan un tratamiento.
a. Lo tomará como le indiquen.
b. Consultará previamente con el nefrólogo.
c. No lo tomará.
d. No sabe.

\section{DIETA}

1. ¿Por qué es importante la dieta?
a. No es importante.
b. Porque determinados alimentos pueden aportar sustancias dañinas para el organismo en el periodo de interdiálisis.
c. Porque una dieta adecuada puede recuperarle la función re- nal.
d. No sabe.

2. ¿Sabe porque son peligrosos los alimentos que contienen potasio?
a. Porque producen picor.
b. Porque producen dolor muscular, parada cardiaca, muerte...
c. Porque producen pérdida de audición.
d. No sabe.

3. ¿Qué alimento contiene más potasio?
a. Verduras y frutas.
b. Leche.
c. Pastas.
d. No sabe.

4. Las verduras son alimentos ricos en potasio. ¿Cómo deben de tomarse para disminuir el nivel de potasio?
a. Crudas.
b. Cocidas.
c. Cocidas en dos aguas.
d. No sabe.

5. ¿Cuántas piezas de fruta puede tomar al día?
a. Ninguna.
b. Una.
c. Todas las que quiera.
d. No sabe.

6. ¿Cuánto líquido puede ingerir en su dieta diaria?
a. Libre.
b. $500 \mathrm{cc}+$ diuresis.
c. Dos litros.
d. No sabe.

7. ¿Qué ocurre cuando ingiere mucho líquido?
a. Respira mal, hinchazón de manos y piernas.
b. Tiene calambres.
c. Orina más.
d. No sabe.

8. ¿Por qué debe de comer sin sal?
a. Para tener menos sed y para que su tensión arterial no se al- tere.
b. Porque se disminuye el tiempo de diálisis.
c. Porque orina más.
d. No sabe. 


\section{FISTULA ARTERIO - VENOSA}

1. ¿Que es una fístula arterio - venosa?
a. Una vena de gran calibre ("gorda")
b. Una arteria
c. La unión de una arteria con una vena para conseguir un flu- jo de sangre elevado al dializarse la vena
d. No sabe.

2. ¿Cómo sabe usted que su físula arterio - venosa funciona?
a. Porque nota thrill ("murmullo") a la palpación
b. Porque nota latido.
c. Porque sus venas están muy engrosadas ("gordas")
d. No sabe

3. ¿Necesita una higiena especial el brazo de la fístula arterio venosa?

a. Sí, cuanto menos moje la zona mejor.

b. Sí, debe utilizar para ello un antiséptico (agua oxigenada, betadice...)

c. Agua y jabón como el resto del cuerpo diariamente.

d. No sabe

4. ¿Puede retirar los apósitos de las zonas de punción de la fístula después de su sesión de hemodiálisis?
a. Sí, inmediatamente
b. No hasta la próxima sesión de diálisis.
c. Sí, pasadas dos horas
d. No sabe

5. En relación al brazo de la fístula arterio - venosa es cierto que:
a. No puede coger pesos, dormir sobre ese brazo, usar prendas apretadas.
b. No puede escribir.
c. Puede realizar cualquier actividad.
d. No sabe

6. En el brazo de la fístula arterio - venosa puede:
a. Tomar la tensión arterial.
b. Dejar extraer sangre para pruebas analíticas o canalizar una vía para sueroterapia.
c. Sólo permitirá manipular la fístula por el personal de diálisis.
d. No sabe

7. En caso de sangrado de su fístula al finalizar la sesión de hemodiálisis o en el período interdialítico:
a. Presiones suavemente sobre el punto de punción mientras se pone en contacto con el servicio de nefrología.
b. Dejarla, parará sola de sangrar.
c. Llamar al médico de cabecera.
d. No sabe

8. Si nota que su fístula arterio - venosa no funciona ¿Qué debe hacer?
a. Lo notificará en la próxima sesión de diálisis.
b. Acudirá al centro lo más rápidamente posible
c. Esperará, porque puede volver a funcionar.
d. No sabe

\section{TRATAMIENTO SUSTITUTIVO / FARMACOLÓGICO}

1. ¿Qué es la hemodiálisis?

a. Es un método de tratamiento de las enfermedades renales para conseguir su curación.

b. Es un método para eliminar toxinas y líquido (que habitualmente se hace mediante el riñón) mediante el paso de dichas toxinas y líquido de la sangre a líquido de diálisis.

c. Es un método que consiste en cambiar la sangre durante la sesión.

d. No sabe

2. Si por algún problema es necesario desechar la sangre del circuito de diálisis:

a. Necesita una transfusión urgente.

b. No pasa nada. El volumen desechado es poca cantidad.

c. Hay que reponer el volumen perdido con suero.

d. No sabe

3. Durante la sesión de hemodiálisis se administra HEPARINA:
a. Para aumentar la velocidad de la circulación de la sangre.
b. Para evitar la coagulación.
c. Para evitar calambres.
d. No sabe

4. ¿Sabe para que sirve la inyección de NESP que se le administra al finalizar la sesión de hemodiálisis?
a. Para aumentar el calcio en los huesos.
b. Para corregir la anemia y evitar transfusiones.
c. Para regular la tensión arterial y la frecuencia cardiaca.
d. No sabe

5. ¿Sabe como debe tomar el RENAGEL, si lo tiene pautado en su tratamiento?
a. Al comienzo de las comidas.
b. Después de las comidas.
c. En medio de las comidas.
d. No sabe

6. ¿Sabe como debe tomar el CARBONATO CALCIO (MASTICAL), si lo tiene pautado en su tratamiento?
a. En medio de las comidas.
b. Antes de las comidas.
c. Después de las comidas.
d. No sabe

7. En relación al líquido que se le extrae en diálisis:
a. Se quita siempre la misma cantidad.
b. Se quita más cantidad al paciente más obeso
c. Si no hay otro dato reseñable se puede eliminar el líquido que ha ganado entre las dos sesiones de hemodiálisis
d. No sabe

8. En relación al FOSFORO:
a. Suele aumentar en la IRC y puede producir picor.
b. Disminuye evitando alimento ricos en fósforo, con la hemo- diálisis y con la medicación.
c. a y b son ciertas
d. No sabe 